Check for updates

Cite this: RSC Adv., 2017, 7, 47373

Received 1st September 2017 Accepted 2nd October 2017

DOI: $10.1039 / c 7 r a 09726 c$

rsc.li/rsc-advances

\section{Large-scale preparation of flexible and reusable surface-enhanced Raman scattering platform based on electrospinning AgNPs/PCL nanofiber membrane $\uparrow$}

\begin{abstract}
Jihua Shi, Tingting You, ${ }^{*}$ Yukun Gao, Xiu Liang, Chenling Li and Penggang Yin (DD*
A flexible and reusable SERS substrates were prepared by electrospinning Ag nanoparticles (AgNPs) in reversed micelle into poly( $\varepsilon$-caprolactone) (PCL) nanofibers, forming nanocomposite membrane with high detection sensitivity. The AgNPs/PCL nanofiber membrane was characterized by scanning electron microscopy (SEM), transmission electron microscopy (TEM) and surface-enhanced Raman scattering spectroscopy (SERS). SEM and TEM image show that AgNPs dispersed homogeneously in PCL nanofibers. The fabricated substrates based on AgNPs/PCL nanofiber membrane have shown reusability and excellent detection reproducibility in SERS under a low concentration of R6G $\left(10^{-12} \mathrm{M}\right)$ and crystal violet $\left(10^{-11} \mathrm{M}\right)$, and the detection limit of melamine is as low as $5 \mathrm{ppb}$, which is far below the safety of FDA and US. Moreover, the substrate showed hydrophobicity and low loss of stress after etching.
\end{abstract}

\section{Introduction}

In recent years, surface-enhanced Raman scattering (SERS) detection has attracted increasing interest due to its abundant applications in the fields of biochemical sensing, photocatalysis, medication and archaeology detection. As a spectroscopic analysis technique, it shows a strong capability of providing structural information from target molecules at a very low concentration, even to single molecule level. ${ }^{1-4}$ Surface plasmon resonance (SPR) properties of noble metal nanoparticles in SERS substrates play a dominant role in the enormous enhancement of the Raman signal of probe molecules, which could enlarge the effective scattering cross section and improve the efficiencies of light harvesting and radiation ${ }^{5-7}$ under an excitation wavelength close to the corresponding SPR band..$^{8-10}$ The preparation of SERS substrates experienced long time development with varied fabrication methods such as chemical vapor deposition, laser deposition, self-assembly etc. Substrate based on electrospinning nanofibers ${ }^{11,12}$ is with larger specific surface area, better flexibility, higher porosity and higher mechanical strength, thus could satisfy the strict requirements for a universal combination strategy ${ }^{13-20}$ and lead to applications in fields as membrane technology, reinforced material and optical sensor.

Key Laboratory of Bio-inspired Smart Interfacial Science and Technology of Ministry of Education, School of Chemistry, Beihang University, Beijing, China.E-mail: pgyin@ buaa.edu.cn

† Electronic supplementary information (ESI) available. See DOI: 10.1039/c7ra09726c
In this paper, the electrospinning technique has been employed to prepare a free-standing, flexible, reproducible and stable AgNPs/PCL nanofiber membrane on a large scale with the introducing of reversed micelle. The obtained substrates possess an extremely long lifetime, reusable and sensitivity in SERS substrates. In our experiments, 4-mercaptophenol (4$\mathrm{Mph}$ ), rhodamine 6G (R6G) and crystal violet have been used as probing molecules. Melamine in milk has also been detected, showing both high efficiency and reproducibility.

\section{Experimental section}

\subsection{Material}

Silver nitrate $\left(\mathrm{AgNO}_{3}\right)$, sodium borohydride $\left(\mathrm{NaBH}_{4}\right)$, trisodium citrate dihydrate $\left(\mathrm{C}_{6} \mathrm{H}_{5} \mathrm{Na}_{3} \mathrm{O}_{7} \cdot 2 \mathrm{H}_{2} \mathrm{O}\right)$, and hydrogen tetrachloroaurate tetrahydrate $\left(\mathrm{HAuCl}_{4} \cdot 4 \mathrm{H}_{2} \mathrm{O}\right)$ were all purchased from Sinopharm Chemical Reagent Co., Ltd. and used as received. Dioctyl sodium sulfosuccinate, R6G, crystal violet and melamine were all purchased from J\&K Scientific Ltd. All solutions were prepared using $18 \mathrm{M} \Omega \mathrm{cm}$ deionized water obtained with a Milli-Q System (Billerica, MA, USA). And the glassware was cleaned by aqua regia $\left(\mathrm{HCl}: \mathrm{HNO}_{3}\right.$ in a $3: 1$ ratio by volume) and rinsed with the deionized water prior to the experiments.

\subsection{Synthesis of AgNPs ${ }^{21}$}

AgNPs were grown using seed-mediated growth. Briefly, a $20 \mathrm{~mL}$ of $1 \%(\mathrm{w} / \mathrm{v})$ citrate solution and were added in flask with $75 \mathrm{~mL}$ water. The mixture was heated to $70{ }^{\circ} \mathrm{C}$ for $15 \mathrm{~min} .1 .7 \mathrm{~mL}$ of $1 \%(\mathrm{w} / \mathrm{v})$ $\mathrm{AgNO}_{3}$ solution was added to the mixture, followed by $2 \mathrm{~mL}$ of $0.1 \%(\mathrm{w} / \mathrm{v})$ freshly prepared $\mathrm{NaBH}_{4}$ solution. The reaction system 
was kept at $70{ }^{\circ} \mathrm{C}$ for $1 \mathrm{~h}$ under continuous stirring and then cooled to room temperature. The resulted production was used as starter seed solution for the following stepwise seeding growth process to obtain larger AgNPs. $6 \mathrm{~mL} \mathrm{1 \%} \mathrm{citrate} \mathrm{solution} \mathrm{was} \mathrm{mixed} \mathrm{with}$ $225 \mathrm{~mL}$ water and kept boiling by a heating mantle for $15 \mathrm{~min}$. $30 \mathrm{~mL}$ of starter seed solution was then added under vigorous stirring, followed by the addition of $5.1 \mathrm{~mL} 1 \% \mathrm{AgNO}_{3}$ solution. The stirring continued for $1 \mathrm{~h}$ before the next seeding growth step was proceeded. Same operation was repeated for two times, resulting in AgNPs with average size of $28.5 \mathrm{~nm}$. For AgNPs of average $45 \mathrm{~nm}$, the resulting $28.5 \mathrm{~nm}$ AgNPs were used as seeds. $6 \mathrm{~mL} \mathrm{1 \%} \mathrm{citrate}$ solution was mixed with $240 \mathrm{~mL}$ water and heated to $80{ }^{\circ} \mathrm{C}$ for $15 \mathrm{~min}$. After that, $30 \mathrm{~mL}$ seed solution was added, followed by the addition of $5.1 \mathrm{~mL} 1 \% \mathrm{AgNO}_{3}$ solution. The reaction system was kept at $80{ }^{\circ} \mathrm{C}$ under continuous stirring for $2 \mathrm{~h}$ and the obtained AgNPs solution was cooled down to room temperature.

\subsection{Preparation of AgNPs/PCL nanocomposite}

The as-prepared AgNPs solution was purified by centrifugation to remove excess sodium citrate and water. The nanoparticles were transferred to a sample vial and dispersed in DMF before it was added into the PCL solution containing Aerosol OT(AOT) micelle under stirring at room temperature. The resulting homogeneous solution was thus obtained for later electrospinning. The mixed solution was loaded into a $10 \mathrm{~mL}$ plastic syringe and electrospun proceeded at positive voltage of $25 \mathrm{kV}$, $12 \mathrm{~cm}$ working distance and $0.2 \mathrm{~mL} \mathrm{~h}^{-1}$ flow rate at room temperature. The resulted AgNPs/PCL nanofiber membrane was finally collected on the aluminum foil.

\subsection{Preparation of SERS sample}

For preparation of SERS substrates, the as-prepared AgNPs/PCL nanofiber membrane was cut into small pieces. $5 \mu \mathrm{L}$ R6G solution was dipped on one piece of the nanocomposites membrane substrate and the sample was directly subjected to measurement with laser Raman spectroscopy. The used SERS substrate was etched with ethanol vapor for 2 min and washed with distilled water. Then the membrane was dried at room temperature and used to as new substrate to detect crystal violet molecule with same method. After a second detection, the membrane was etched again with ethanol vapor for $2 \mathrm{~min}$ to remove the adsorbed probe molecules and used to detect melamine in milk in the third time.

\subsection{Treatment of milk sample with melamine}

Milk samples were prepared following the procedures of the reported method. ${ }^{25}$ Briefly, melamine standard aqueous solution was diluted in mile with different concentration $(100 \mathrm{ppm}$, $10 \mathrm{ppm}, 1 \mathrm{ppm}, 100 \mathrm{ppb}, 25 \mathrm{ppb}, 5 \mathrm{ppb}) .2 .0 \mathrm{~mL}$ of diluted solution sample was dissolved in $2.0 \mathrm{~mL} 1.0 \mathrm{M}$ trichloroacetic acid (in water) and mixed by using the ultrasonic cleaner for $10 \mathrm{~min}$ to precipitate proteins. The mixture was then centrifuged at $8000 \mathrm{rpm}$ for $10 \mathrm{~min}$, and $3 \mathrm{~mL}$ of the supernatants were purified with solid phase extraction. The extracting solution was dried under $\mathrm{N}_{2}$ atmosphere and redissolved in $1.5 \mathrm{~mL}$ deionized water, which could be used for further experiments.

\subsection{Characterization}

SEM characterizations were performed with JSM-7500F field emission scanning electron micro-analyzer. TEM images were recorded on JEM-2100 transmission electron microscope. Raman scattering spectra was recorded with LabRAM HR800 spectrometer using the $514 \mathrm{~nm}$ excitation. UV-vis-NIR spectra were recorded on UV-3600 at room temperature. The mechanical properties of the membrane were tested using the HYSITRON TI-950 Tribo Indenter. A Berkovich shape indenter was used.

\section{Result and discussion}

\subsection{Preparation of AgNPs/PCL nanofiber membrane}

AgNPs were prepared by aqueous synthesis and the seedmediated Lee-Meisel method. ${ }^{21}$ AgNPs with size distribution of $45.2 \pm 2.6 \mathrm{~nm}$ are prepared as shown in Fig. 1a. The UV-vis absorption spectrum of the AgNPs aqueous dispersion shows that the particles have a characteristic surface plasmon resonance (SPR) wavelength at $428 \mathrm{~nm}$ (Fig. 1b). The XRD spectra of AgNPs was shown in Fig. S1. $\dagger$ The synthesized AgNPs were directly used in the subsequent electrospinning process.

The synthetic strategy for fabricating AgNPs/PCL nanofibers is shown in Fig. 2. The AgNPs were washed with distilled water and then concentrated with DMF (dimethyl formamide). Then the solution was added to the as-prepared PCL solution containing AOT (sodium bis(2-ethylhexyl) sulfosuccinate) under stirring overnight. Finally, AgNPs/PCL nanofibers were obtained by electrospinning of the PCL aqueous solution with AgNPs nanocomposites.

The reverse micelle technology is adopted to make AgNPs disperse in the organic solvents, while electrospinning technique is applied to stabilize the assembled AgNPs within the polymer nanofibers. Collected on an aluminum mesh with a woven structure, the composite nanofibers are aligned on a large scale. As shown in Fig. 3, the nanofibers successfully produced by electrospinning are with threedimensional network structure consisting of a large quantity of randomly deposited fibers. The hydrophilic AgNPs are assembled within the nanofibers, which were stable and dispersive due to the impact of reverse micelles. The average diameter length of Ag/PCL nanofibers are about $800 \mathrm{~nm}$. The backscattering SEM image and TEM image in Fig. 3 and 4 both indicated that AgNPs aggregates were embedded in PCL matrix with chain-like distribution along the axial direction. High applied static voltage led to polarization of solution. The PCL-capped and reverse micelle capsulated AgNPs became positively charged on one end and negatively charged on the other. Under electrostatic absorption, the polarized AgNPs tended to form a chain-like distribution oriented along the electric filed in the blend solution. When the fluid filament was squeezed from the jet, AgNPs aggregates were embedded in PCL nanofibers with the solvents evaporated. In the following section, AgNPs/PCL nanofiber with molar ratio of $203: 5$ between PCL repeat units and Ag atoms will be discussed. 

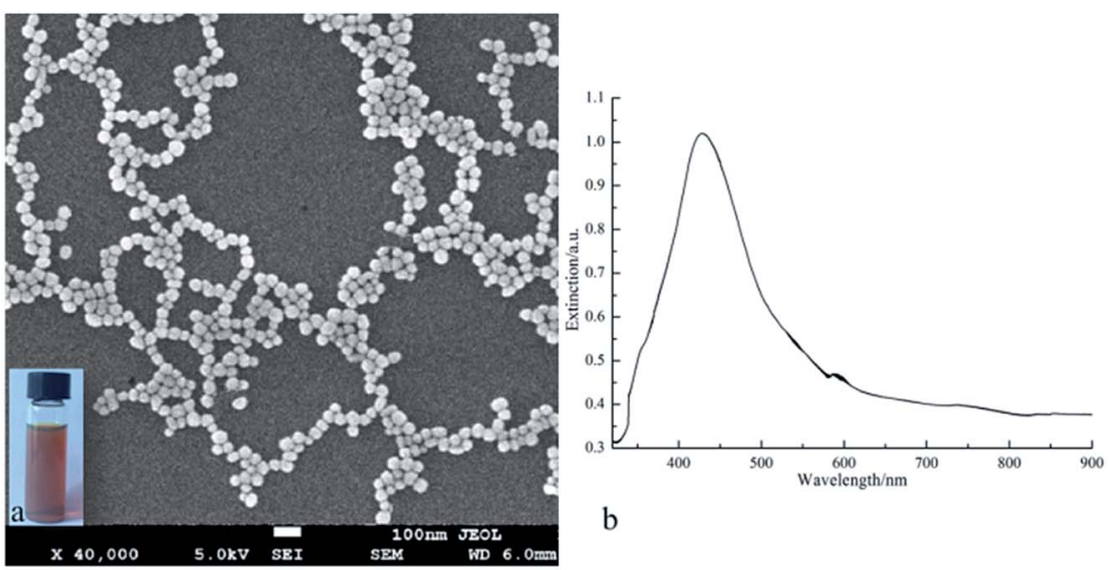

$\mathrm{b}$

Fig. 1 SEM image (a) and UV-vis spectrum (b) of the prepared AgNPs, inset in (a) is photograph of the AgNPs colloids.

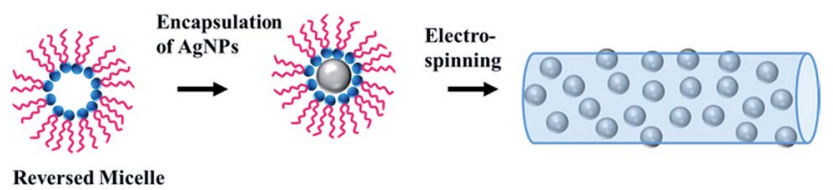

$\underset{\sim}{\sim} \longrightarrow$ AOT O $\longrightarrow$ AgNP $\square \longrightarrow$ PCL Nanofiber

Fig. 2 Schematic illustration for the preparation of AgNPs/PCL nanocomposite membrane.

\subsection{Surface-enhanced Raman scattering characterization}

Compared with normal rigid SERS substrates, flexible membrane substrates can be easily tailored and wrapped around nonplanar surfaces. The assembled AgNPs aggregates in PCL fibers could induce great enhancement in local electromagnetic field, making it excellent SERS substrates as low cost integrated sensors for daily life and practical applications with high sensitivity. Fig. S2 $\uparrow$ shows the Raman spectrum of as-prepared AgNPs/PCL nanofiber membrane. No pronounced signals are found for substrates without probe molecules. SERS behavior of several analytes absorbed on the nanocomposite substrates were tested, including R6G, crystal violet, 4 -Mph and melamine. $5 \mu \mathrm{L}$ aqueous solution of the analyte was directly dripped onto the surface of the AgNPs/PCL nanofiber membrane. The SERS samples were then subjected to Raman spectroscopy measurements with a $50 \times$ objective (the spot size is about $1 \mu \mathrm{m}$ ) under $514 \mathrm{~nm}$ excitation (the laser power is $0.5 \mathrm{~mW}$ ). Fig. 5 shows a set of SERS spectra of the AgNPs/PCL nanocomposite membrane with different concentrations of 4-Mph at an excitation wavelength of $514 \mathrm{~nm}$. The observed Raman bands could be assigned to ring-breathing
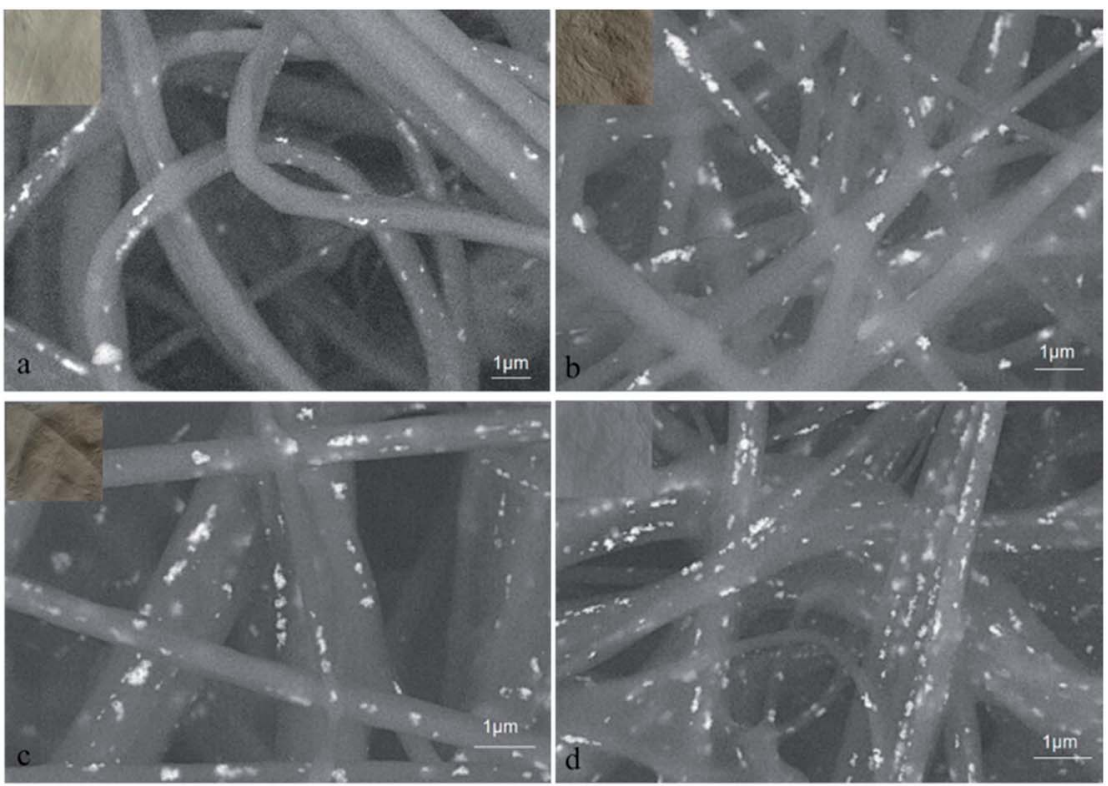

Fig. 3 Backscattering SEM image of AgNPs/PCL nanofiber membrane with different molar ratio of AgNPs (in terms of Ag atoms) to PCL (in terms of repeat units): (a) $203: 1$, (b) $203: 3$, (c) $203: 4$, (d) $203: 5$. Insert is the photograph of the corresponding AgNPs/PCL nanofiber membrane. 

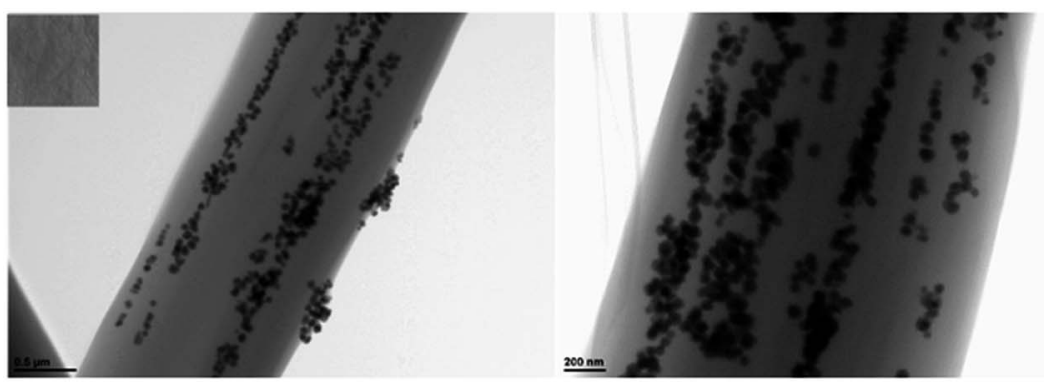

Fig. 4 TEM image of AgNPs/PCL nanofiber membrane with a AgNPs : PCL molar ratio of $203: 5$. Insert is the photograph of the corresponding AgNPs/PCL nanofiber membrane.

modes $\left(993 \mathrm{~cm}^{-1}\right)$ and $\mathrm{C}-\mathrm{C}$ stretching modes $\nu(\mathrm{C}-\mathrm{C})_{\text {ring }}$ $\left(1593 \mathrm{~cm}^{-1}\right), \mathrm{C}-\mathrm{S}$ stretching modes $\nu(\mathrm{C}-\mathrm{S})\left(1086 \mathrm{~cm}^{-1}\right)$ and $\mathrm{C}-\mathrm{H}$ in-plane bending modes $\beta(\mathrm{C}-\mathrm{H})_{\text {ring }}\left(1173 \mathrm{~cm}^{-1}\right)$ of $4-\mathrm{Mph}$ molecules. High quality Raman spectra shown in Fig. 5 indicate an satisfactory SERS performance of the fabricated substrates. After the detection of 4-Mph, the used AgNPs/PCL nanofiber membrane substrates were etched by hot ethanol and dried under $\mathrm{N}_{2}$ atmosphere. Then the processed substrate was used directly for R6G molecules detection.

Surface enhanced Raman spectra for R6G absorbed on the processed AgNPs/PCL nanofiber membrane were shown in Fig. 6. Distinctive peaks were observed and assigned as $\mathrm{C}-\mathrm{C}$ inplane deformation modes $\delta(\mathrm{C}-\mathrm{C})_{\text {ring }}\left(613 \mathrm{~cm}^{-1}\right)$, C-H out of plane deformation modes $\delta(\mathrm{C}-\mathrm{H})\left(775 \mathrm{~cm}^{-1}\right), \mathrm{C}-\mathrm{H}$ and $\mathrm{N}-\mathrm{H}$ bending modes $\beta(\mathrm{C}-\mathrm{H})_{\mathrm{xanthene}}$ and $\beta(\mathrm{N}-\mathrm{H})_{\mathrm{xanthene}}\left(1186 \mathrm{~cm}^{-1}\right.$ and $\left.1224 \mathrm{~cm}^{-1}\right), \mathrm{C}=\mathrm{C}$ stretching modes $\nu(\mathrm{C}=\mathrm{C})_{\text {xanthene }}$ $\left(1312 \mathrm{~cm}^{-1}\right)$, C-C stretching modes $\nu(\mathrm{C}-\mathrm{C})_{\mathrm{xanthene}}\left(1513 \mathrm{~cm}^{-1}\right.$ and $\left.1583 \mathrm{~cm}^{-1}\right)$ and $\mathrm{C}=\mathrm{O}$ stretching modes $\nu(\mathrm{C}=\mathrm{O})$ $\left(1650 \mathrm{~cm}^{-1}\right)$. Homogeneity of the spectral signal over a large area is of great importance when considering the SERS substrates in practical application. Thus, a mapping measurement was conducted via spot to spot Raman spectra on a $20 \times$ $20 \mu \mathrm{m}^{2}$ area of $10^{-10} \mathrm{M}$ R6G absorbed on the shallow etched

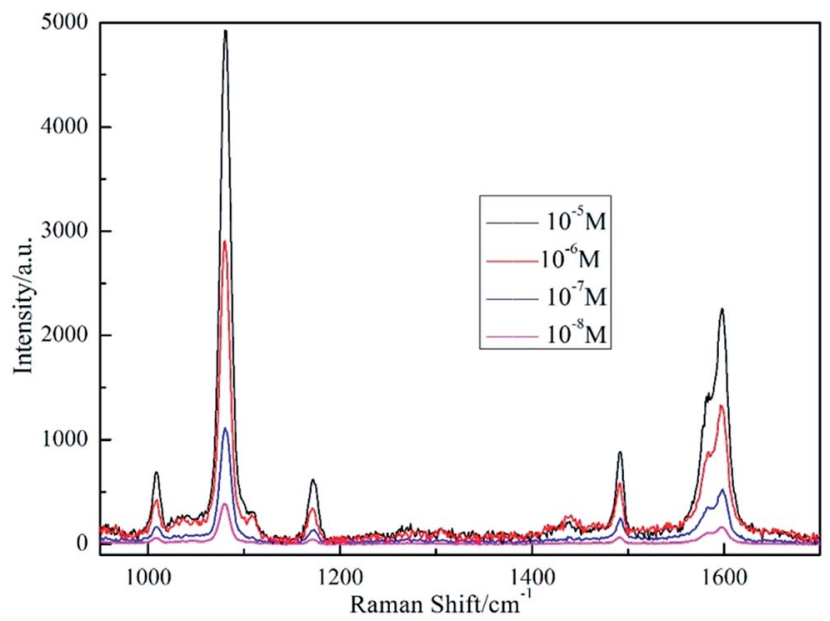

Fig. 5 SERS spectra of 4-Mph absorbed on AgNPs/PCL nanofiber membrane with different 4-Mph concentrations of $10^{-5} \mathrm{M}, 10^{-6} \mathrm{M}$, $10^{-7} \mathrm{M}$ and $10^{-8} \mathrm{M}$, respectively. The acquisition time is $3 \mathrm{~s}$.
AgNPs/PCL nanofiber membrane with a step size of $1 \mu \mathrm{m}$ to evaluate the substrate reproducibility. As shown in Fig. 7, the relative standard deviation (RSD) was calculated to be $7.78 \%$ for Raman band at $1650 \mathrm{~cm}^{-1}$, indicating good uniformity in large area. Measurement results suggest that the AgNPs/PCL nanofiber membrane could be a good candidate for label free detection with high sensitivity and reproducibility. The enhanced SERS signals mainly came from R6G molecules absorbed on the hot spots of the substrates, such as gaps between AgNPs. The uneven and irregular structure as seen from Fig. S4† also improves the sensitivity of the fabricated nanocomposite membrane. As characterized by Fig. $\mathrm{S} 3, \uparrow$ the etched substrates also exhibits certain hydrophobicity.

Following the detection of R6G probe molecules, the same AgNPs/PCL nanofiber membrane substrate was etched again by hot ethane. The SEM image of the double etched substrate was shown in Fig. S4(c). $\dagger$ After drying the processed substrates at $\mathrm{N}_{2}$ atmosphere, $5 \mu \mathrm{L}$ crystal violet solutions was dropped onto the nanocomposite membrane. Surface enhanced Raman spectra for crystal violet was collected and shown in Fig. 8. Distinctive peaks were observed and assigned to characteristic vibrational modes of crystal violet molecules including C-phenyl deformation modes $\delta\left(\mathrm{C}\right.$-phenyl) $\left(333 \mathrm{~cm}^{-1}\right)$, aromatic nucleus bending

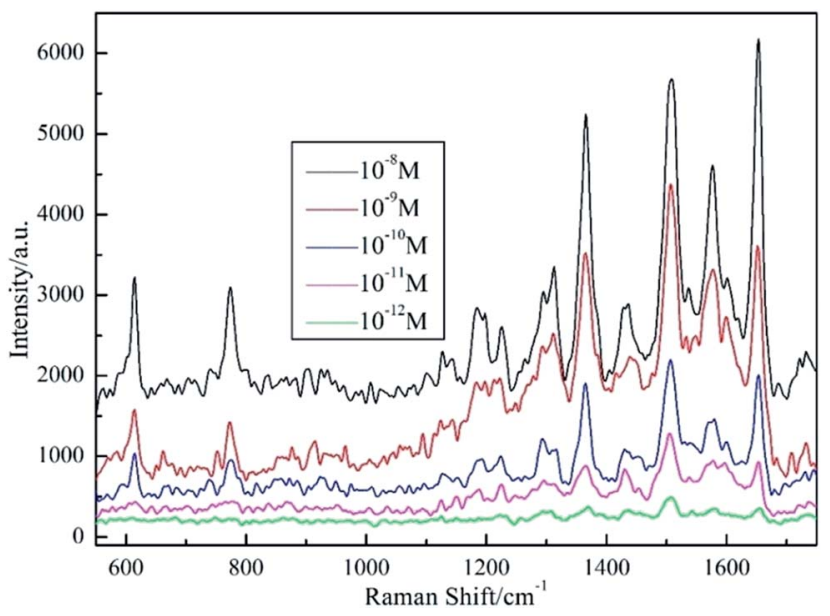

Fig. 6 SERS spectra of R6G absorbed on AgNPs/PCL nanofiber membrane with different R6G concentrations of $10^{-8} \mathrm{M}, 10^{-9} \mathrm{M}$, $10^{-10} \mathrm{M}, 10^{-11} \mathrm{M}$ and $10^{-12} \mathrm{M}$, respectively. Acquisition time is $3 \mathrm{~s}$. 

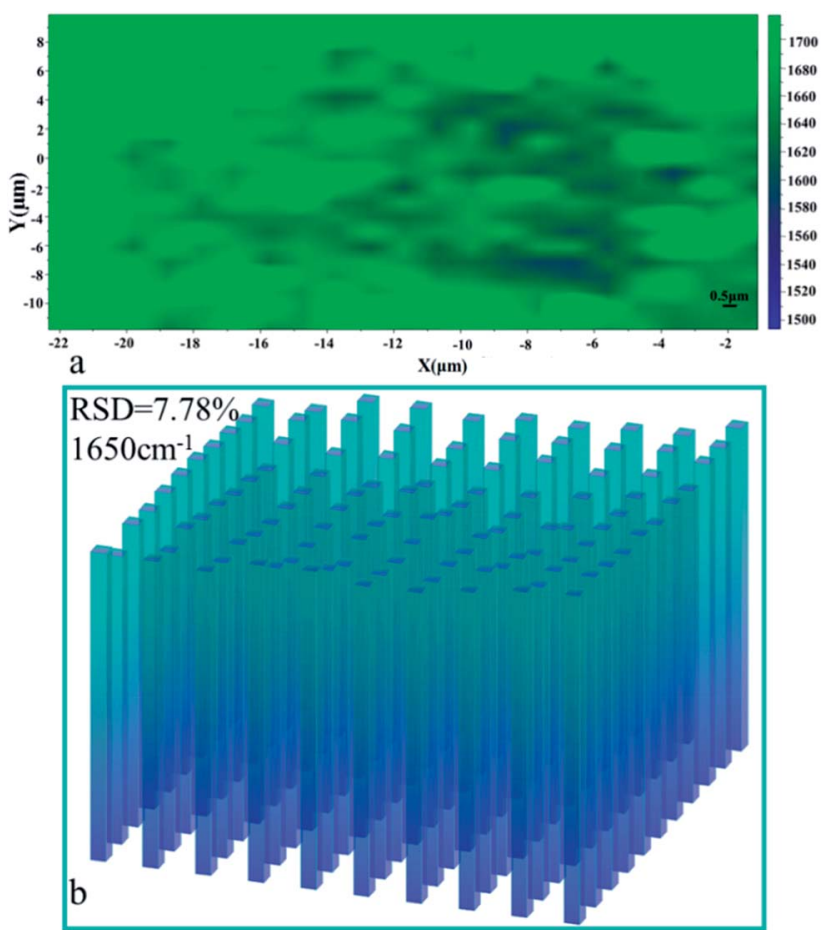

Fig. 7 (a) Raman intensity maps at $1650 \mathrm{~cm}^{-1}$ of $10^{-10}$ M R6G molecules on a $20 \times 20 \mu \mathrm{m}^{2}$ surface area of AgNPs/PCL nanofiber membrane; (b) the corresponding intensity distributions at $1650 \mathrm{~cm}^{-1}$ of R6G molecules in (a).

modes $\gamma$ (aromatic nucleus) $\left(420 \mathrm{~cm}^{-1}\right)$, C-phenyl bending modes $\gamma\left(\mathrm{C}\right.$-phenyl) $\left(441 \mathrm{~cm}^{-1}\right)$, radial aromatic ring skeleton stretching modes $\nu$ (radial aromatic ring skeleton) $\left(525 \mathrm{~cm}^{-1}\right.$, $558 \mathrm{~cm}^{-1}$ and $\left.913 \mathrm{~cm}^{-1}\right), \mathrm{C}-\mathrm{H}$ bending modes $\gamma(\mathrm{C}-\mathrm{H})$ $\left(723 \mathrm{~cm}^{-1}, 765 \mathrm{~cm}^{-1}\right.$ and $\left.802 \mathrm{~cm}^{-1}\right)$, C-H deformation modes $\delta(\mathrm{C}-\mathrm{H})\left(1174 \mathrm{~cm}^{-1}\right), \mathrm{C}-\mathrm{C}$ stretching modes $\nu_{\mathrm{s}}(\mathrm{C}-\mathrm{C})_{\text {aromatic ring }}$ (1296 $\left.\mathrm{cm}^{-1}\right), \quad \mathrm{N}-$ phenyl stretching modes $\nu$ (N-phenyl) $\left(1385 \mathrm{~cm}^{-1}\right)$, C-C stretching modes $\nu_{\mathrm{s}}(\mathrm{C}=\mathrm{C})_{\text {aromatic }}$ ring

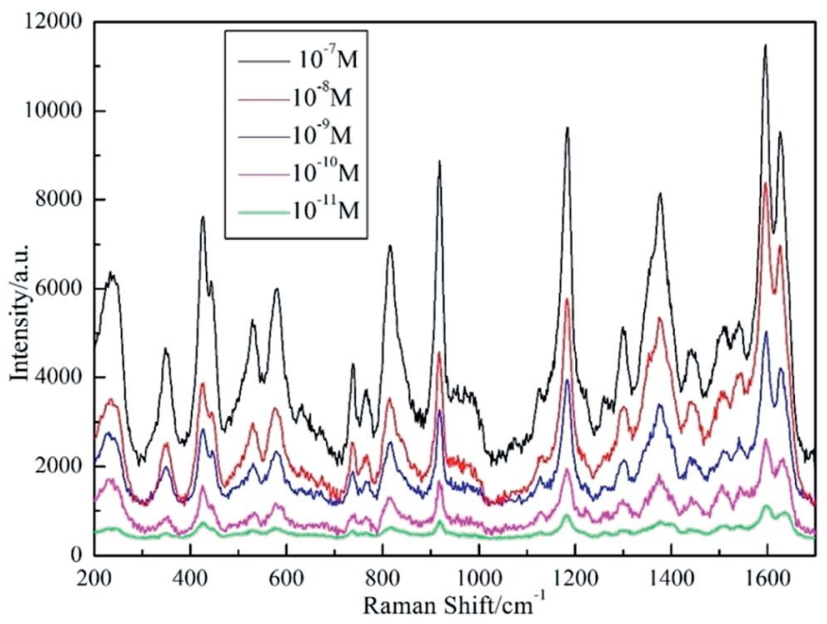

Fig. 8 SERS spectra of $C V$ absorbed on AgNPs/PCL nanofiber membrane with different $\mathrm{CV}$ concentrations of $10^{-7} \mathrm{M}, 10^{-8} \mathrm{M}$, $10^{-9} \mathrm{M}, 10^{-10} \mathrm{M}$ and $10^{-11} \mathrm{M}$, respectively. Acquisition time is $4 \mathrm{~s}$. $\left(1592 \mathrm{~cm}^{-1}\right)$ and $\nu_{\text {as }}(\mathrm{C}=\mathrm{C})$ aromatic ring $\left(1620 \mathrm{~cm}^{-1}\right)$. There were no signals observed for R6G molecules in Fig. 8, verifying the reused ability of the fabricated AgNPs/PCL nanofiber membrane. To prove the homogeneity of the double etched substrates, a mapping measurement was conducted via spot to spot Raman spectra on a $40 \times 36 \mu \mathrm{m}^{2}$ area of $10^{-10} \mathrm{M}$ crystal violet on the shallow etched AgNPs/PCL nanocomposite substrates with a step size of $1 \mu \mathrm{m}$. As shown in Fig. 9, the relative standard deviation was calculated to be $8.23 \%$ for Raman band at $1592 \mathrm{~cm}^{-1}$, indicated good uniformity of the substrate in a large area.

\subsection{SERS detection of trace melamine}

Rapid detection and identification of poisonous substances in food or environment is one of the most important tasks for SERS analysis. Melamine is an organic chemical material and is mainly used to produce melamine-formaldehyde resins for glues, adhesives, and plastics. However, due to its low cost and high nitrogen content ( $66 \%$ by mass), melamine has been illegally added to dairy products to boost the apparent protein contents. ${ }^{22}$ The safety limits of melamine in food are $2.5 \mathrm{ppm}$ in the United States and $1 \mathrm{ppm}$ in China. ${ }^{23}$ SERS spectra melamine on as-prepared substrates are recorded using LabRAM HR800 spectrometer with $514 \mathrm{~nm}$ excitation. The Raman spectrum of melamine samples is shown in Fig. 10. The typical Raman peaks

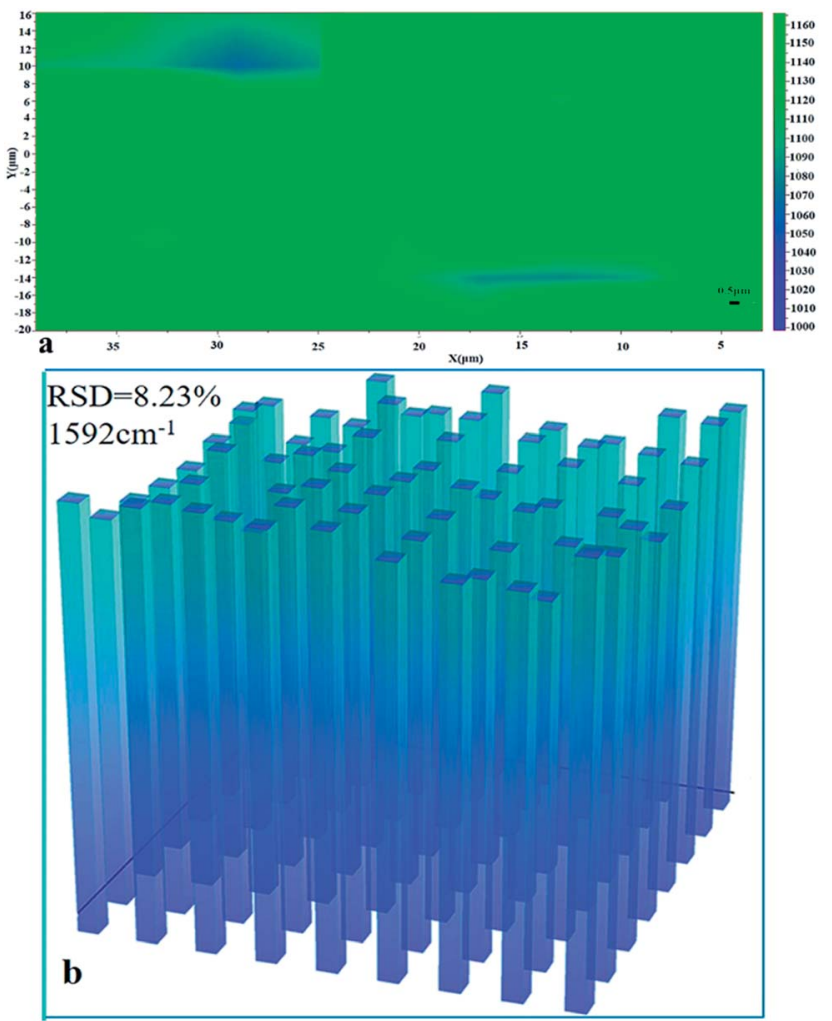

Fig. 9 (a) Raman intensity maps at $1592 \mathrm{~cm}^{-1}$ of $10^{-10} \mathrm{M} \mathrm{CV}$ molecules on a $40 \times 36 \mu^{2}$ surface area of AgNPs/PCL nanofiber membrane; (b) the corresponding intensity distributions at $1592 \mathrm{~cm}^{-1}$ of CV molecules in (a). 


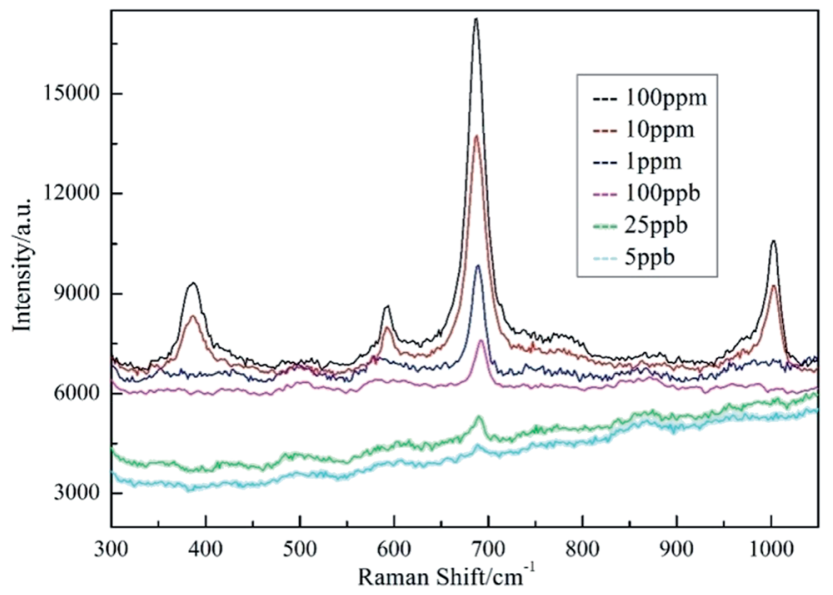

Fig. 10 SERS spectra of AgNPs/PCL nanofiber membrane with different melamine concentrations. The acquisition time is $5 \mathrm{~s}$.

of melamine are at 380, 581, 686 and $987 \mathrm{~cm}^{-1}$. The strongest Raman peak at $686 \mathrm{~cm}^{-1}$ belongs to the ring breathing II mode related to the in-plane deformation of the triazine ring. Raman peak at $987 \mathrm{~cm}^{-1}$ belongs to the ring breathing I mode of the triazine ring. ${ }^{24}$ When the concentration of melamine is lower than $100 \mathrm{ppb}$, the characteristic peak of melamine at $686 \mathrm{~cm}^{-1}$ could still be observed, indicating the high sensitivity of AgNPs/ PCL nanofiber membrane substrate in practical detection.

\subsection{Mechanical properties}

In this paper, we report a free-standing AgNPs/PCL nanofiber membrane substrate fabricated on a large scale. To prove the free-standing performance, the mechanical property of the fabricated membrane was tested. Typical stress-strain curves of AgNPs/PCL nanofiber membrane are presented in Fig. 11. The tensile strength of the sample before etching is $20 \mathrm{MPa}$ and the value illustrates that the nanocomposite membrane is completely self-supported. Both tensile strength and strain

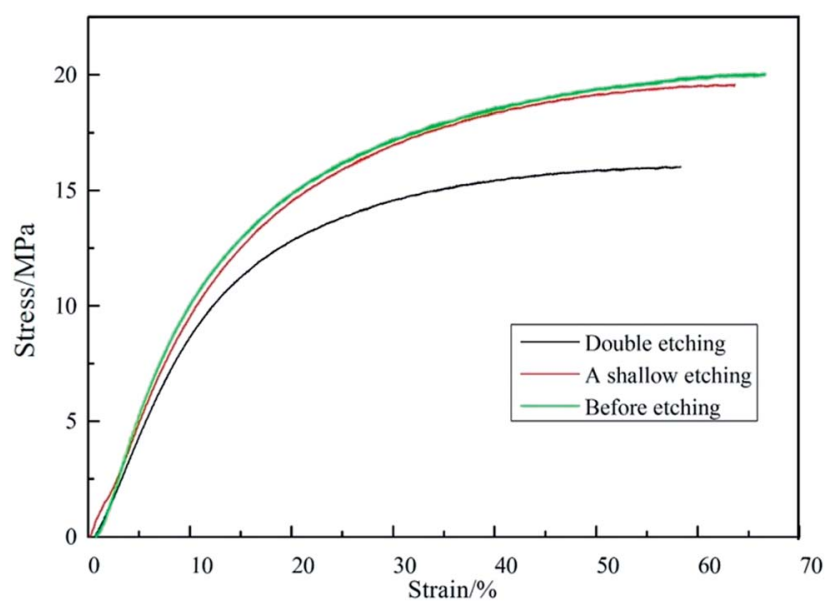

Fig. 11 Tensile stress-strain curves of the AgNPs/PCL nanofiber membrane before etching (green line), after one shallow etching (red line) and after double etching (black line). decreased with the elapsing of time during etching process, but the rate of decreasing is relatively small. The substrates with double etching could still be considered as self-supported according to the measurements, indicating good reproducibility in addition to high flexibility and mechanical strength.

\section{Conclusion}

To fabricate a reusable, flexible SERS substrate with high sensitivity, AgNPs in reversed micelle were electrospun into PCL nanofibers, forming nanofiber membrane with nobel nanoparticle aggregates. The successfully prepared AgNPs/PCL nanofiber membrane in this work have shown excellent detection sensitivity under a low concentration of R6G $\left(10^{-12} \mathrm{M}\right)$ and crystal violet $\left(10^{-11} \mathrm{M}\right)$. The average RSD values of the major Raman peak are all less than 0.09. The detection limit of melamine is as low as $5 \mathrm{ppb}$, which is far below the safety limitation of FDA and US. R6G, CV and melamine were detected on the same piece of AgNPs/PCL nanofiber membrane which was etched after every detection, indicating good reproducibility of the substrate. Further study illustrates that the substrate also exhibited hydrophobicity and low loss of stress after etching, opening up new strategy to the routine SERS detection for a wide range of application.

\section{Conflicts of interest}

There are no conflicts to declare.

\section{Acknowledgements}

This work was supported by the National Natural Science Foundation of China (51572009 and 51272013).

\section{References}

1 S. Rupp, A. Off, H. Seitzmoskaliuk, et al., Sensors, 2015, 15, 23110-23125.

2 A. F. Palonpon, J. Ando, H. Yamakoshi, et al., Nat. Protoc., 2013, 8, 677-692.

3 P. Lovera, N. Creedon, H. Alatawi, et al., Nanotechnology, 2014, 25, 912908-912909.

4 L. Yang, P. Li, H. Liu, et al., Chem. Soc. Rev., 2015, 44, 28372848.

5 C. L. Zhang, K. P. Lv, H. P. Cong, et al., Small, 2012, 8, 647653.

6 W. Tang, D. B. Chase and J. F. Rabolt, Anal. Chem., 2013, 85, 10702-10709.

7 L. Zhang, X. Gong, Y. Bao, et al., Langmuir, 2012, 28, 1443314440.

8 C. Marega, J. Maculan, R. G. Andrea, et al., Nanotechnology, 2015, 26, 598-602.

9 C. L. Zhang, K. P. Lv, H. T. Huang, et al., Nanoscale, 2012, 4, 5348-5355.

10 W. Gao, G. Chen, W. Xu, et al., RSC Adv., 2014, 4, 2383823845.

11 D. He, B. Hu, Q. F. Yao, et al., ACS Nano, 2009, 3, 3993-4002. 
12 K. Kim, J. Y. Choi, H. B. Lee, et al., ACS Appl. Mater. Interfaces, 2010, 2, 1872-1878.

13 X. Li, M. Cao, H. Zhang, et al., J. Colloid Interface Sci., 2012, 382, 28-35.

14 Y. Bao, C. Lai, Z. Zhu, et al., RSC Adv., 2013, 3, 8998-9004.

15 Y. Wang, Q. Yang, G. Shan, et al., Mater. Lett., 2005, 59(24), 3046-3049.

16 T. Szymborski, E. Witkowska, W. Adamkiewicz, et al., Trop. Med. Int. Health, 2014, 139, 5061-5064.

17 V. Fasano, M. Baroncini, M. Moffa, et al., J. Am. Chem. Soc., 2014, 136, 14245-14254.

18 N. Liu, K. Kim, P. C. Hsu, et al., J. Am. Chem. Soc., 2014, 136, 17284-17291.
19 Y. Qian, G. Meng, Q. Huang, et al., Nanoscale, 2014, 6, 47814788.

20 C. L. Zhang, K. P. Lv, H. P. Cong, et al., Small, 2012, 8, 647653.

21 Y. Wan, Z. Guo, X. Jiang, et al., J. Colloid Interface Sci., 2013, 394, 263.

22 M. Thompson, L. Owen, K. Wilkinson, R. Wood and A. Damant, Analyst, 2002, 127, 1666-1668.

23 X. S. Liang, H. P. Wei, et al., Analyst, 2011, 136, 179-183.

24 R. Li, G. Yang, J. Yang, et al., Food Control, 2016, 68, 14-19.

25 L. Li, B. X. Li, D. Cheng and L. H. Mao, Food Chem., 2010, 122, 895-900. 